\title{
Rotational Speed Measurement Based on Laser Mouse Sensors
}

\author{
Cheng Liu', Yanan Xu'/2, Ji-Gou Liu', Hui Sun ${ }^{1}$, Ralph Kennel ${ }^{2}$ \\ ${ }^{1}$ ChenYang Technologies GmbH \& Co. KG, Markt Schwabener Straße 8, 85464 Finsing, Germany \\ ${ }^{2}$ Institute for Electrical Drive Systems and Power Electronics, Technische Universität München, \\ Arcisstraße 21, 80333 Munich, Germany \\ Emails: cheng.liu@chenyang-ism.com; john.liu@cy-sensors.com; ralph.kennel@tum.de
}

\begin{abstract}
In this paper, a rotational speed measuring method is proposed on the basis of a laser mouse sensor. A target disc, which reflects the laser beam, is mounted on the shaft of a motor under test. The laser mouse sensor is coupled with the target disc with an air gap. The change of the air gap between the laser sensor and target disc can be sampled with the laser mouse sensor ADNS-9800 of Avago/PixArt, so that the rotational speed from 50 RPM to 6000 RPM can be determined by using Fast Fourier Transform. The laser mouse sensor PLN-2037 of Philips uses displacement counts in order to determine the tangential speed, which is proportional to the rotational speed. This sensor can be used for measuring the speed from 10 RPM to 50 RPM. A combination of both sensors ADNS9800 and PLN-2037 will provide a measuring range from 10 RPM to 6000 RPM.
\end{abstract}

Keywords: Rotational Speed, Electrical Drives, Servo Motor, Laser Mouse Sensor, Laser Interferometry

\section{Introduction}

Rotational speed measurement is an important operation for applications in industry and automation. Especially in electrical drive systems, rotational speed sensors are essential for a proper functionality of the whole machine [1].

In the past, analogue precision tachometers or tachogenerators did a quite good job for drive systems [2]. Nowadays the commonly used rotational speed sensors in electrical drive systems are mainly optical and magnetic encoders. For some high precision applications, encoders with high resolution and accuracy are needed. However, these encoders are very expensive.

A better solution is to develop a cheaper rotational speed sensor with comparable accuracy like high resolution encoders. Laser mouse sensors are widely used in high performance mice for computer gamers. They are cheap in production and can reach high resolution up to 8200 DPI (Dots Per Inch). So it is motivated to develop a rotational speed measuring system based on laser mouse sensors.

Two common laser mouse sensors ADNS9800 of Avago/Pixart and PLN-2037 of Philips are considered. Both sensors provide a resolution of $8200 \mathrm{DPI}$, but their working principles are different. The sensor ADNS-
9800 is based on the Laser-Speckle interferometry and CMOS imaging technology [3], while the sensor PLN-2037 works according to the Laser-Doppler effect [4].

\section{Working Principle of ADNS-9800}

The laser mouse sensor ADNS-9800 offers a resolution up to $8200 \mathrm{CPI}$, a moving speed up to 150 IPS (Inches Per Second), a processing speed of 12000 FPS (Frames Per Second) and a maximum acceleration up to $30 \mathrm{G}$ [3].

The sensor is based on the Laser-Speckle interferometry and CMOS imaging technology. The sensor package contains a vertical-cavity surface-emitting laser (VCSEL), the sensor based on CMOS or CCD technology, an imaging lens and a collimating lens (see Fig. 1).

By optically acquiring sequential surface images (known as frames) via an Image Acquisition System (IAS), a Digital Signal Processor (DSP) can calculate the displacement by comparing the contrast of two images in a row (see Fig. 2). So the corresponding displacement in $X$ and $Y$ directions of the surface can be determined mathematically by the DSP [6]. By using Serial Peripheral Interface (SPI), the DSP can establish a communication interface with an external microcontroller for sensor data output. 


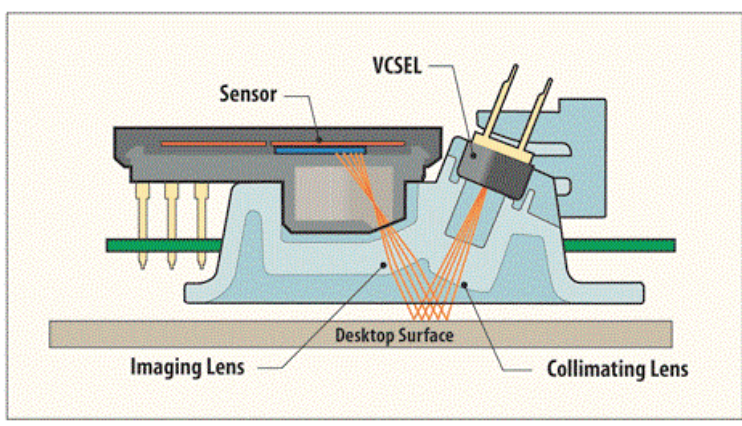

Fig. 1: Internal Structure of an ADNS-9800 Laser Mouse Sensor [5].

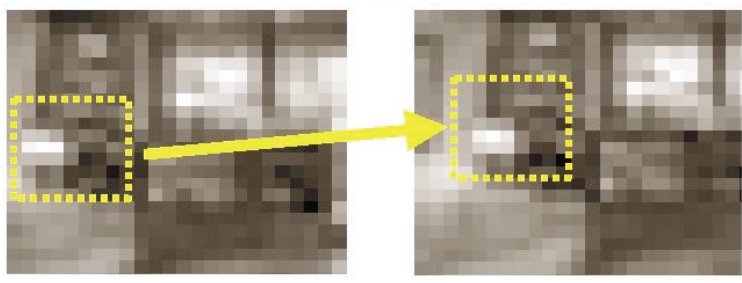

Fig. 2: Working Principle of an ADNS-9800 Laser Mouse Sensor [5].

\section{Working Principle of PLN-2037}

The working principle of the PLN-2037 is based on the principle of Laser Doppler Effect, which also uses a VSCEL as its laser source. As the Fig. 3 shows, the laser is aimed at a moving object in a distance of $d / 2$ and in an angle of $\phi$. A small part of the scattered light from the moving surface is reflecting back into the laser source where it interferes with the original laser field. This procedure is called Laser Self-Mixing Interferometry ([7], [8]).

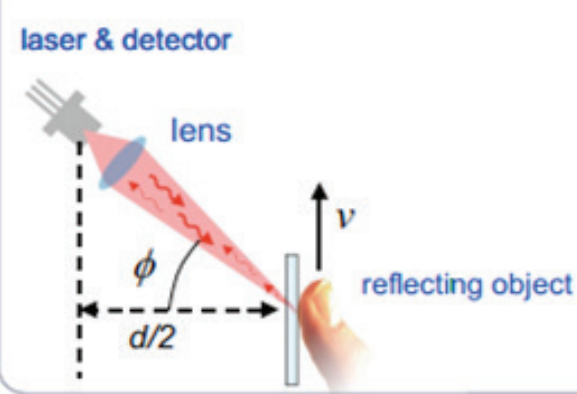

Fig. 3: $\quad$ Working Principle of PLN-2037 Laser Mouse Sensor ([4], [8]).

With the laser beam having a component in the same direction as the movement of the object, the phase of the reflected light continuously shifts with respect to the original laser light, resulting in a periodic variation of the output signal from the detector. The frequency of this output signal is equal to the Doppler frequency, according to the following formula:

$$
f_{\text {Doppler }}=\frac{2 v \cdot \cos \phi}{\lambda}
$$

where $v \cdot \cos \phi$ is the velocity component, which is along the direction of the laser beam, and $\lambda$ is the wavelength of the laser beam. The Doppler frequency is linear to the moving velocity.

Therefore, the object velocity can be calculated by determining the Doppler frequency, for example with the Fast Fourier Transformation (FFT) [9].

The direction of the motion can also be detected by adding a frequency modulation technique. The result is a frequency shift of $f_{0}$, which is called the undulation frequency. When the object is moving, the Doppler frequency will influence the undulation frequency, depended on the moving direction:

$$
\begin{aligned}
& f_{\text {up }}=f_{0}-f_{\text {Doppler }} \\
& f_{\text {down }}=f_{0}+f_{\text {Doppler }}
\end{aligned}
$$

By using (1) to (3), both the amplitude and the direction of the object velocity can be determined.

Using the Laser Self-Mixing Interferometry, the laser mouse sensor PLN-2037 obtains the following specifications: resolution up to 8200 $\mathrm{CPI}$, a moving speed up to 150 IPS (Inches Per Second) and a maximum acceleration up to $50 \mathrm{G}$ [4].

\section{Test Systems}

For measuring the rotational speed, a rotational speed measuring system is built with an ADNS-9800, a microcontroller unit (MCU) and a Host-PC. Fig. 4 shows the functional block diagram of this system.

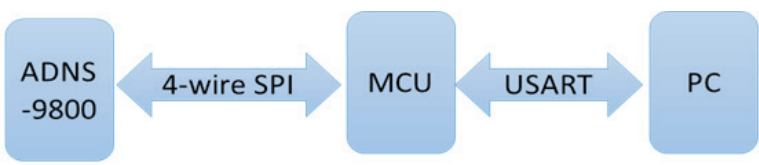

Fig. 4: Functional Block Diagram of the Rotational Speed Measuring System with ADNS-9800 [5].

The communication between ADNS-9800 is realized with a four-wire SPI connection, while the MCU communicates with the Host-PC through a serial port connection USART and RS-232.

The ADNS-9800 is mounted on a U-shaped plate above the target disc driven by a servo 


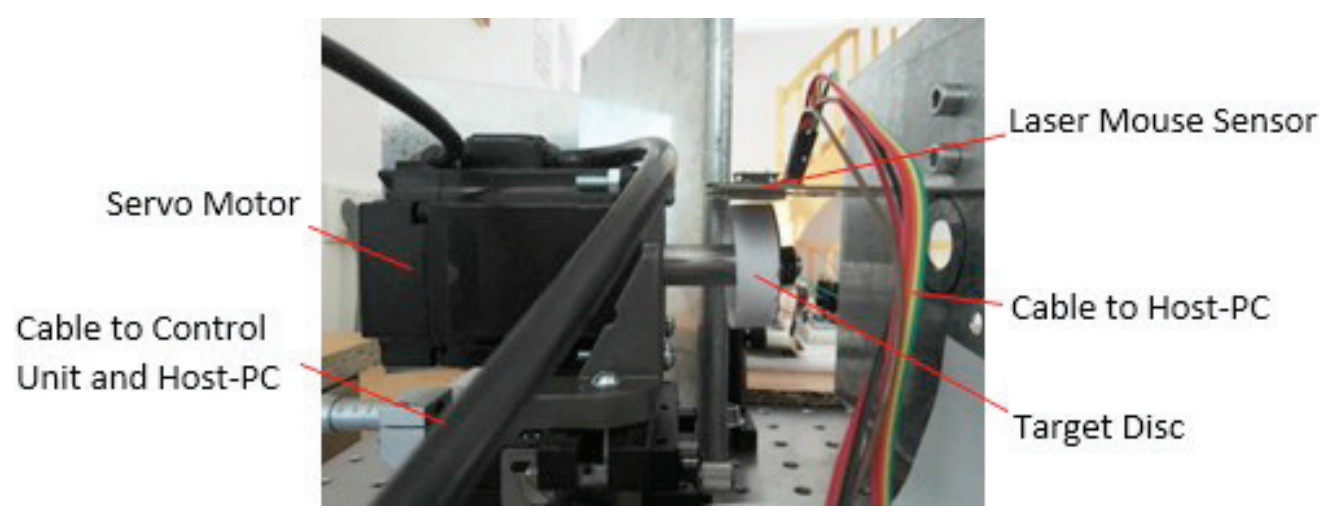

Fig. 5: Rotational speed of a servo motor is measured with a Laser Mouse Sensor ADNS-9800.

motor, see Fig. 5. Therefore, the laser mouse sensor can measure the displacement from the target disc.

The rotational speed of the servo motor can be set through the Host-PC by software. Here the rotational speed reference is given by the speed data from the 24-bit encoder built in the servo motor.

For testing the PLN-2037 of Philips, the gaming mouse Naga 2014 of Razer Inc. is taken into account. The Razer Naga 2014 contains the PLN-2037 as its navigation sensor. The highest DPI setting is 8200 , which results in a displacement resolution of $3.1 \mu \mathrm{m}$. The laser mouse sensor is accompanied with a 32-bit ARM processor. The microprocessor has the task to read the data from the sensor, to compute the corresponding displacement counts and send them to the PC. By using some Windows API functions provided by Microsoft, it is possible to read the displacement counts from the mouse in pixels and calculate the corresponding displacement in metric units $(\mu \mathrm{m}, \mathrm{mm}, \mathrm{cm}$ etc.). The mouse sends the displacement information to the computer at a certain rate, the so-called polling rate or report rate. The highest USB polling rate of this mouse is $1000 \mathrm{~Hz}$, which means, the mouse sends displacement information every $1 \mathrm{~ms}$. The following block diagram in Fig. 6 shows the functionality of displacement determination with Razer Naga 2014 in the PC system.

In order to ensure the circuitry function of the Razer Naga 2014, the mouse is tested directly. Fig. 7 shows the test system, which consists of the same servo motor and target disc like in the ADNS-9800 test system (Fig. 5).

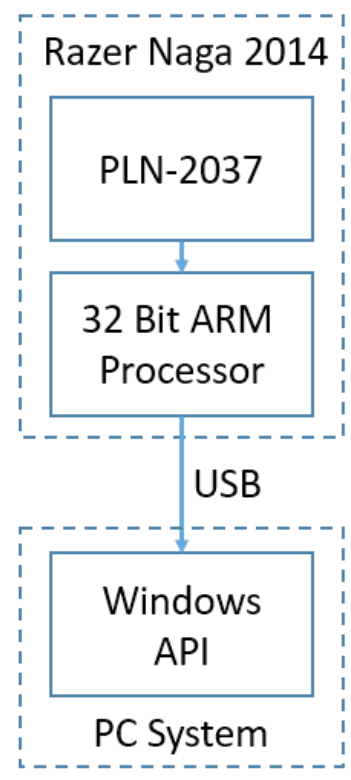

Fig. 6: Functional Block Diagram of the Rotational Speed Measuring System with PLN-2037.

The Razer Inc. provides the configuration tool 'Synapse' for the Naga 2014 laser gaming mouse [13]. With this tool, the resolution of the mouse can be set, and the surface can be calibrated. Concretely, the mouse can be adjusted to its best performance on different surfaces. Experiments indicated that a white and rough surface provides the best performances. So the mouse is calibrated on white paper, which wraps up the target disc. With the software tool, the liftoff range can also be set, so that the maximum distance between the bottom of the mouse and the object surface can be optimized. In our tests, this range is set to $3 \mathrm{~mm}$. 


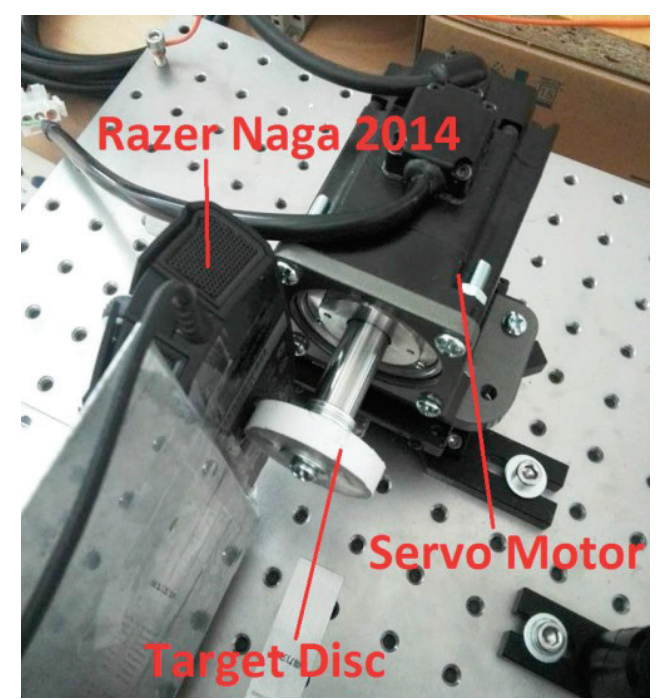

Fig. 7: Rotational speed of a servo motor is measured with a Laser Mouse Razer Naga 2014, which includes the Laser Mouse Sensor PLN-2037.

\section{Test Results}

Due to an eccentricity of the target disc mounted on the motor shaft, the sensor ADNS9800 gives out a sinusoidal output signal (see Fig. 8), as a result of the periodic air gap change between sensor and target disc. By applying a Fast Fourier Transform (FFT), the fundamental frequency and consequently the rotational speed can be determined. The PLN2037 isn't affected by this eccentricity, so that the rotational speed is calculated from the displacement counts and the diameter of the target disc.

With the ADNS-9800, good results are achieved in the speed range from 50 RPM to 6000 RPM (see Fig. 9). The relative errors are controlled within $0.5 \%$, so that this sensor is preferred for measurements of high rotational speeds.

In contrast, the PLN-2037 shows better results in low speeds. In the measuring range from 10 RPM to 160 RPM, the errors are less than 3\% (see Fig. 10). It is possible to get a better measuring accuracy $(<1.0 \%)$ by optimizing the sensor structure.

From the measurement results, it can be concluded that both sensors are complementary. Therefore, a novel rotational speed sensor with both sensors ADNS-9800 and PLN-2037 will provide good accuracy within $1.0 \%$ in the speed range from 10 RPM to $50 \mathrm{RPM}$ and $0.5 \%$ in the speed range from 50 RPM to 6000 RPM.

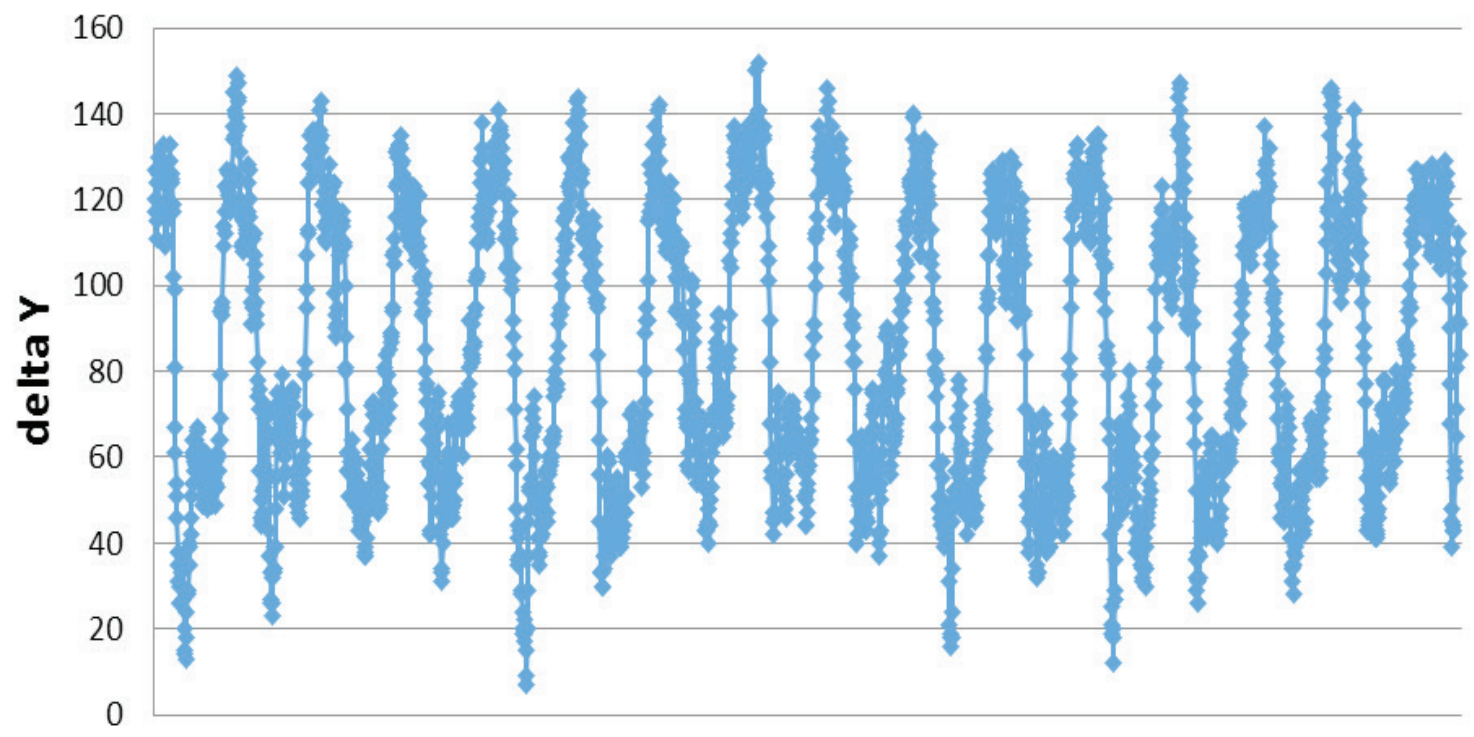

Fig. 8: Sensor Signal Measured at 100 RPM with ADNS-9800 [5]. 


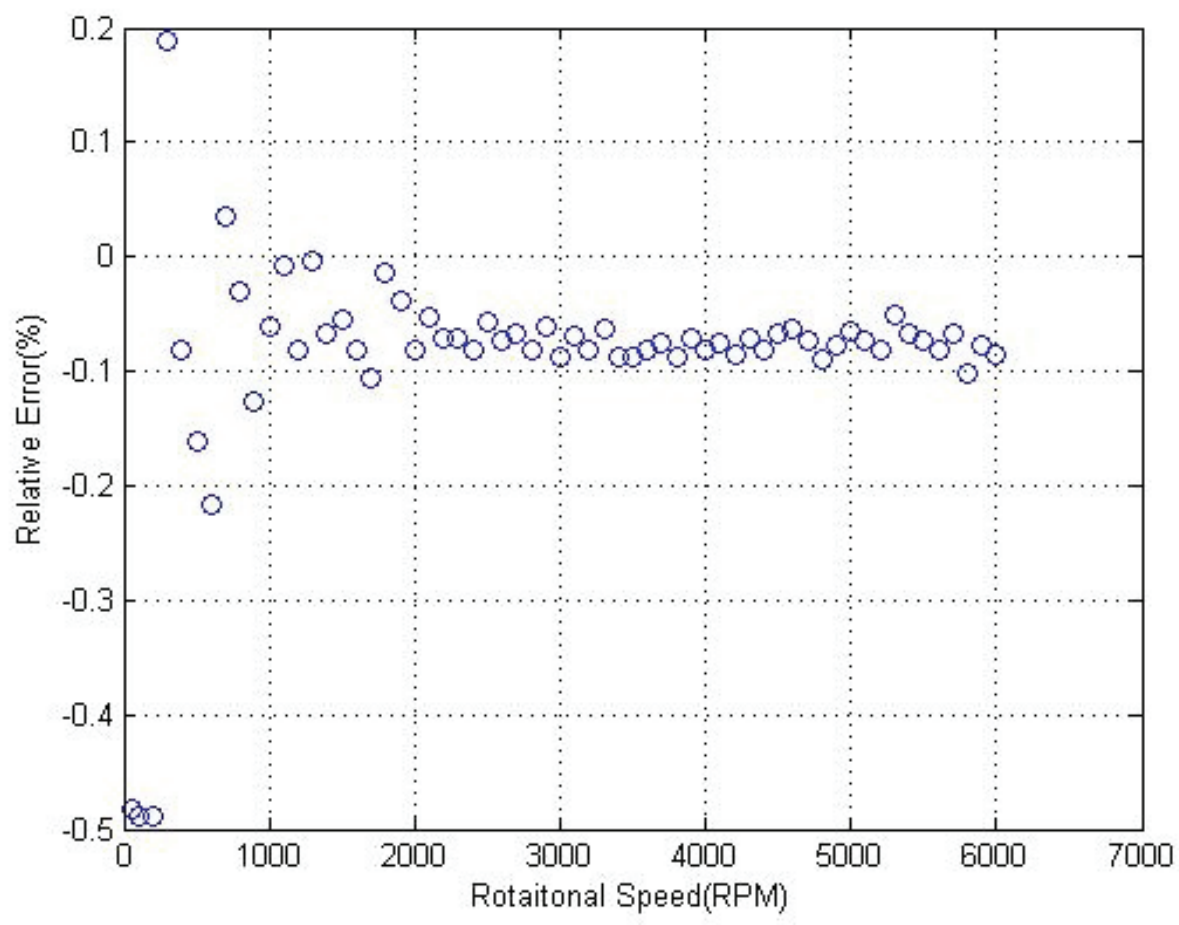

Fig. 9: Relative Errors of the Rotational Speed Measurement from 50 RPM to 6000 RPM with ADNS-9800 [5].

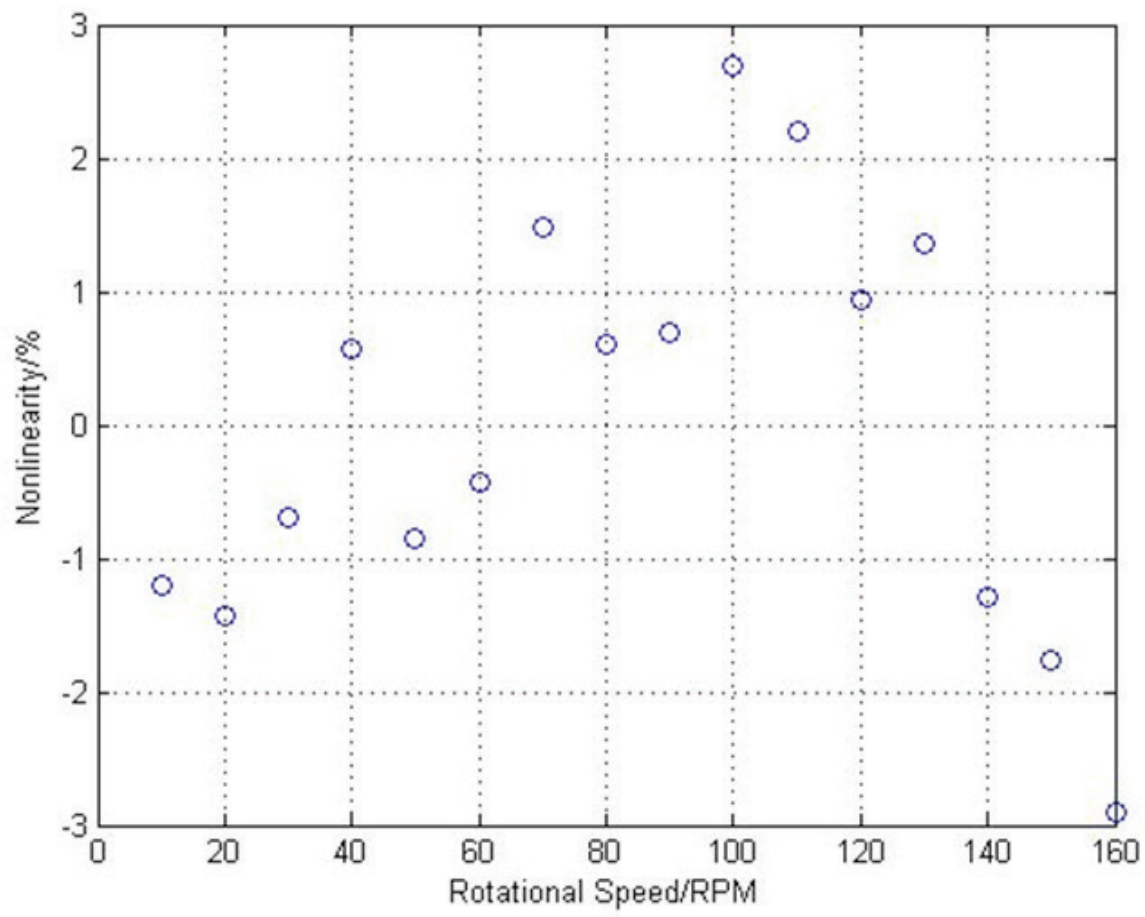

Fig. 10: Relative Errors of the Rotational Speed Measurement from 10 RPM to 160 RPM with PLN-203 [5]. 


\section{Conclusions}

This paper presents rotational speed measuring systems which are based on laser mouse sensors ADNS-9800 and PLN-2037.

From the results one can draw the following conclusions:

- The laser mouse sensor ADNS-9800 works with the Laser Speckle and Image Acquisition Principle. Two images are taken in a very short time and compared towards each other in order to determine the displacement.

- $\quad$ The laser mouse sensor PLN-2037 works according to the Laser Self-Mixing Interferometry. The measurand is the Doppler Frequency, which can be extracted from the scattered laser light of the moving surface.

- Both laser Mouse sensors provide nearly the same resolution (8200 CPI) and moving speed (150 IPS), and can be used for rotational speed measurement.

- By measuring the air gap change of the rotational target disc, the laser mouse sensor ADNS-9800 gives out a periodic output signal, which comprises the fundamental frequency and therefore the rotational speed. A measuring accuracy of $0.5 \%$ is achieved for the range from 50 RPM to 6000 RPM.

- The displacement information of the laser mouse sensor PLN-2037 can be read by a Windows API in a PC system. In this way, rotational speed can be determined. The accuracy of this method after optimization is less than $1.0 \%$ in the range from 10 RPM to 160 RPM.

- The measurement range of both laser mouse sensors is complementary, so that rotational speed sensors can be developed by using both laser mouse sensors.

Further research works should be done in order to improve the measuring accuracy of the rotational speed sensors by optimization of the sensor parameters.

\section{References}

[1] D. Schröder, Elektrische Antriebe - Regelung von Antriebssystemen, 2nd Edition, SpringerVerlag, Berlin, Heidelberg, New York, 2001.

[2] R. Kennel, "Why Do Incremental Encoders Do a Reasonably Good Job in Electrical Drives with Digital Control?", 41th IEEE IAS 2006 Annual Meeting, Tampa, Florida, 2006.

[3] PixArt Imaging Inc., Datasheet ADNS-9800.
[4] Philips Photonics, PLN-2037 Product Specification.

[5] Y. Xu, Study of Rotational Speed Measurement Based on Laser Mouse Sensor with Applications to Electric Driving Systems, Master Thesis, Technische Universität München, ChenYang Technologies $\mathrm{GmbH}$ \& Co. KG, August 27, 2015.

[6] B. A. Wandell A. El Gamal, B. Girod, Common Principles of Image Acquisition Systems and Biological Vision, Proceedings of the IEEE, Vol. 90, No. 1, January 2002.

[7] G. Giuliani, M. Norgia, S. Donati, T. Bosch, Laser diode self-mixing technique for sensing applications, Journal of Optics A: Pire Appl. Opt. 4 (2002), S283 -S294.

[8] A. Pruijmboom, M. Schemmann, J. Hellmig, J. Schutte, H. Moench, J. Pankert, VCSEL-based miniature laser-Doppler interferometer, Proc. of SPIE Vol. 6908 69080I-1.

[9] A. Pruijmboom, S. Booij, M. Schemmann, K. Werner, P. Hoeven, H. van Limpt, S. Intemann, R. Jordan, T. Fritzsch, H. Oppermann, M. Barge, VCSEL-based miniature laser-selfmixing interferometer with integrated optical and electronic components, Proc. of SPIE Vol. 7221 72210S-1.

[10] P. Drabarek, R. Kennel, "Are Interferometric Encoders a Reasonable Alternative in Servo Drive Applications?", PEMD 2008, IET 4th International Conference on Power Electronics, Machines \& Drives, York, United Kingdom, Apr. 2008.

[11] L. E. Drain, The Laser Doppler Technique, John Wiley \& Sons, 1980.

[12]E. Schrüfer, Elektrische Messtechnik Messung elektrischer und nichtelektrischer Größen, 6th Edition, Carl Hanser Verlag, Munich, Germany, 1995.

[13] Razer Inc., Razer Naga 2014 Gaming Mouse Product Specification.

[14] C. Liu, J.-G. Liu, R. Kennel, A Rotational Speed Measuring and Calibration System based on Laser Doppler Velocimetry, XXI IMEKO World Congress "Measurement in Research and Industry", August 30 - September 4, 2015, Prague, Czech Republic.

[15] E. Schrüfer, Signalverarbeitung: Numerische Verarbeitung digitaler Signale, 1st Edition; Carl Hanser Verlag, Munich, Germany, 1990. 